\title{
Bildungswärmen der Oxyde von Vanadium und Uran.
}

\author{
Von \\ W. G. MrXTEE. ${ }^{1}$ \\ Mit 1 Figur im Text.
}

Die Bestimmung der Bildungswärmen der Oxyde von Vanadium ist eine einfache kalorimetrische Arbeit, vorausgesetzt, daß das reine Metall in Form eines feinen Pulvers zugänglich ist. Dies konnte nicht erbalten werden, und die Ergebnisse zeigen nur, daß die Oxydationswärme sehr hoch ist.

\section{Vanadium.}

ROSCOE erhielt ziemlich reines metallisches Vanadium in kleinen Mengen durch Reduktion des Chlorids mit Wasserstoff; Morssan reduzierte das Oxyd mit Kohle im elektrischen Ofen, wobei er ein Produkt erhielt, das mehrere Prozent Kohlenstoff enthielt. Andere Methoden sind wohl versucht worden, haben aber mit einer später zu erwähnenden Ausnahme nur unreines Metall geliefert. Fine als elektrolytisches Vanadium verkaufte Probe enthielt nur $85 \%$ des Metalles und eine andere als "geschmolzen, chemisch rein" bezeichnete $82 \%$. In beiden Proben ist Kohle enthalten. Weiss und ArCHEL $^{2}$ reduzierten Vanadiumpentoxyd mit Mischmetall und erhielten ein geschmolzenes, metallisches Produkt, das brüchig war und Quarz ritzte. Zur Analyse verwandelten sie $0.2062 \mathrm{~g}$ daron in Vanadylsulfat und füllten die Lösung auf $500 \mathrm{ccm}$ auf. Sie titrierten $20 \mathrm{ccm}$ der Lösung, die $0.0082 \mathrm{~g}$ enthielten und fanden $0.0082 \mathrm{~g}$ Vanadium. Es ist zweifelhaft, ob eine mit so kleinen Mengen ausgeführte Analyse genaue Resultate liefert. Der Ver. fasser hat zur Bestimmung des Vanadiums die folgende ältere Methode benutzt: $0.2-0.5 \mathrm{~g}$ des Metalles oder Oxyds werden in Salpetersäure gelöst und die Lösung in einem gewogenen Platintiegel trocken gedampft und langsam in einem elektrischen Ofen auf eine

1 Aus dem Àmer. Journ. Sci. (Sill.) ins Deutsche übertragen von I. KopperBerlin.

2 Lieb. Ann. 337, 380.

Z. anorg. Chem. Bd. 78. 
Temperatur erwärmt, die etwas unter dem Schmelzpunkte von Vanadiumpentoxyd liegt. Die Erhitzung wiederholte man, bis konstantes Gewicht erreicht war. Man fand, daB beim Befeuchten des Rückstandes mit Salpetersäure, Trocknen und abermaligen Erhitzen das Gewicht nicht zunahm. Man prüfte die Methode durch Behandeln von $0.5740 \mathrm{~g}$ Vanadiumpentoxyd mit Salpetersüure; das Gewicht blieb unverändert. Das Verfahren setzt voraus, daß keine Stoffe vorhanden sind, die einen Rückstand hinterlassen, oder daß man diese gesondert bestimmt.

Man fand, daß Magnesium beim Erhitzen mit einem Vanadiumoxyd heftig reagiert. In der Hoffnung, das Metall oder ein niedriges Oxyd in Pulverform zu erhalten, führte man den folgenden Versuch aus. Ein inniges Gemisch von $23 \mathrm{~g}$ Vanadiumpentoxyd und $19 \mathrm{~g}$ Magnesiumpulver wurde in einer schweren Messingbombe erhitzt. Unterhalb Rotglut setzte die Reaktion ein und die Bombe wurde rotglühend. Das poröse braune Produkt wurde mit einer Lösung von Ammoniumchlorid behandelt, die das vorhandene Magnesium auflöste, nicht jedoch das Vanadat oder Vanadit. Sodann wusch man das Produkt, trocknete und pulverisierte es und digerierte es hierauf wiederholt mit sehr verdünnter Salzsäure und schließlich mit Säure, die mit dem doppelten Volumen Wasser verdünnt war. In diesem Stadium bestand das Produkt aus Körnern mit metallischem Glanz und einem braunen Pulver. Dieses schlemmte man ab, und den Rückstand wusch man und trocknete ihn im luftrerdünnten Raume über Schwefelsäure. Es war ein Gemisch von glänzenden, metallartigen Teilchen und einem grauen Pulver. Es wurde langsam durch heiße konzentrierte Salzsäure unter Entwickelung eines Gases angegriffen, aber nicht völlig gelöst. Man bezeichnete es mit „A“; die Analyse lieferte folgende Ergebnisse:

\begin{tabular}{|c|c|c|c|}
\hline & I & II & Mittel \\
\hline Vanadium . & 90.1 & 90.0 & 90.0 \\
\hline Magnesiumoxyd . . & 1.44 & 1.49 & $1 . \tilde{5}$ \\
\hline
\end{tabular}

Das Aton:verhältnis von Vanadium zu Sauerstoff ist $3.3: 1$.

Demnächst wurde ein Gemisch von $38 \mathrm{~g}$ Vanadiumtrioxyd und $22 \mathrm{~g}$ Magnesium in einer Stahlbombe erhitzt und eine Zeitlang bei Kirschrotglut gehalten. Dann kühlte man die Bombe unter Wasser ab, um Oxydation ihres Inhaltes zu vermeiden. Das Produkt digerierte man mit heißer, konzentrierter Chlorwasserstoffsäure, wusch es einmal, und dann ließ man es einen Tag in verdünnter Säure. 
Es wurde schließlich gewaschen und wie gewöhnlich getrocknet. Das Produkt wog $18 \mathrm{~g}$ und enthielt $85.2 \%$ Vanadium. Man behandelte es nochmals mit heiber, konzentrierter Salzsäure, wobei sich viel Wasserstoff entwickelte. SichlieBlich wusch man den Rück. stand mit Alkohol und trocknete ihn im luftverdünnten Raume. Er wog $12 \mathrm{~g}$ und wurde mit „B“ bezeichnet. Eine Analyse ergab $84.5 \%$ Vanadium und $0.2 \%$ Magnesiumoxyd. Nachdem das Präparat 2 Wochen gestanden hatte, fand man $83.4 \%$ Vanadium. Vanadiummonoxyd, $\mathrm{V}_{2} \mathrm{O}$, enthält $86.4 \%$ Metall.

Ein weiteres, als "C" bezeichnetes Präparat wurde wie die oben beschriebenen $18 \mathrm{~g}$ hergestellt und enthielt $89.7 \%$ Vanadium. Es war ein dunkelgraues Pulver, das unter dem VergröBerungsglas keine metallischen Teilchen zeigte. Das durch Magnesium reduzierte Metall ist ein außerordentlich feines Pulver, das sich nur langsam in Wasser absetzt und zur Oxydation sehr geeignet ist. Das oxydierte Produkt jedoch scheint ein Gemisch zu sein, denn es ist zum Teil in Salzsäure löslich unter Fntwickelung eines Gases, das ohne Zweifel Wasserstoff ist.

Die folgenden Bestimmungen wurden ausgeführt mit dem Präparat „A“, das 90\% Vanadium und $8.5 \%$ Sauerstoff enthielt.

Versuch 1. $1.000 \mathrm{~g} \mathrm{~A}, 1.000 \mathrm{~g}$ Schwefel, $15 \mathrm{~g}$ Natriumperoxyd. Wärmetönung 9003 cal. Zieht man 5270 cal für Schwefel und 30 cal für verbranntes Eisen, sowie 71 cal für absorbierten Sauerstoff $a b$, so bleiben 3632 cal für die Wärmetönung der Reaktion von $1 \mathrm{~g}$ unreinem Vanadium mit Natriumperoxyd.

Versuch 2 wurde wie Nr. 1, aber mit $1.200 \mathrm{~g}$ "A" ausgeführt. Ergebnis für $1 \mathrm{~g} 3721 \mathrm{cal}$.

Die Verbrennungen waren offenbar vollständig, da die Schmelzen beim Auflösen in Wasser keine unverbrannten Partikeln zurückließen. Das Mittel der Resultate ist 3677 cal. Teilt man diese Zabl durch 0.9 - die Vanadiummenge in $1 \mathrm{~g} \mathrm{-}$, so erhält man 4086 cal und für die Reaktionswärme von $102 \mathrm{~g}$ Vanadium 2 Grammatomen - mit Natriumperoxyd 416800 cal. Aus dieser Zabl leitet man $\mathrm{ab}: 2 \mathrm{~V}+5 \mathrm{O}=\mathrm{V}_{2} \mathrm{O}_{5}+348000$ cal. Wenn man eine Korrektion für den Sauerstoff im benutzten Metall einführt, indem man die Wärmetönung von 1 Atom zu 145000 cal setzt, so wird das Resultat 422000 cal. Präparat „B“ gab bei einem Versuche mit derselben Korrektion 460000 cal. Der Mittelwert der beiden letzten Zablen ist $441000 \mathrm{cal}$. 
Roscok ${ }^{1}$ gibt an, daß Vanadiummonoxyd, $\mathrm{V}_{2} \mathrm{O}$, gebildet wird durch längeres Verweilen von fein rerteiltem Vanadium an der Luft, aber er sagt nichts über die Kigenschaften des Produktes, ausgenommen, daB es ein braunes Pulver ist. Er gibt auch an, daB Vanadium nicht von Chlorwasserstoffsäure angegriffen wird. Ich habe gefunden, daB alle Präparate, die durch Reduktion mit Magnesium gewonnen sind, Salzsäure mit Wasserstoff entwickeln und dabei eine grüne Lösung und einen grauen Rückstand liefern, der sich nicht beim langen Erhitzen auf $100^{\circ}$ in konzentrierter Säure im zugeschmolzenen Rohr löst. Die Entwickelung von Wasserstoff ist offenbar nicht bedingt durch die Einwirkung von metallischem Vanadium auf die Säure, sondern durch das Monoxyd oder Dioxyd, die unter Bildung von $\mathrm{V}_{2} \mathrm{Cl}_{2}$ (unbekannt) oder $\mathrm{VCl}_{2}$ reagieren, welche beide aus Chlorwasserstoff Wasserstofl frei machen. RosCoE ${ }^{2}$ gibt an, daß bei Zusatz von Salzsäure zu einer violetten Lösung von Vanadiumdichlorid Wasserstoff entwickelt wird unter Bildung von Trichlorid.

\section{Vanadiumpentoxyd.}

Vanadiumpentoxyd wurde in üblicher Weise hergestellt durch Errhitzen von Ammoniumvanadat. Dies Präparat wird gewöhnlich als amorph betrachtet, aber unter dem Mikroskop erweist es sich als kristallinisch. Es mag sein, daß die Form der Kristalle von Ammoniumvanadat beim Erhitzen nicht geändert wird, und daB die Zwischenrïume nicht sichtbar sind. Das mit "D" bezeichnete Präparat war ziemlich rein, wie die folgenden Angaben zeigen: $10.413 \mathrm{~g}$ verloren beim Erhitzen auf schwache Rotglut in Wasserstoff $1.8300 \mathrm{~g}$ oder $17.61 \%$, während man für die Umwandlung von $\mathrm{V}_{2} \mathrm{O}_{5}$ in $\mathrm{V}_{2} \mathrm{O}_{3} 17.58 \%$ berechnet. Das bei diesem Versuche erhaltene Vanadiumtrioxyd wird mit „,F" bezeichnet. Ein Teil von $\mathrm{D}$ wurde in einer Platinschale geschmolzen, die rote kristallisierte Probe bezeichnete man mit ,E“.

Die kalorimetrischen Versuche 3 und 4 fübrte man mit dem gelbbraunen, sogenannten amorphen Muster "D" und 5 und 6 mit dem roten kristallisierten Präparat "E“ aus. Folgendes sind die Zahlen und Ergebnisse.

1 Roscoe-Schorlemmer, A Treatise on Chemistry, 4. Aufl., II, 902.

2 1. C., S. 909. 


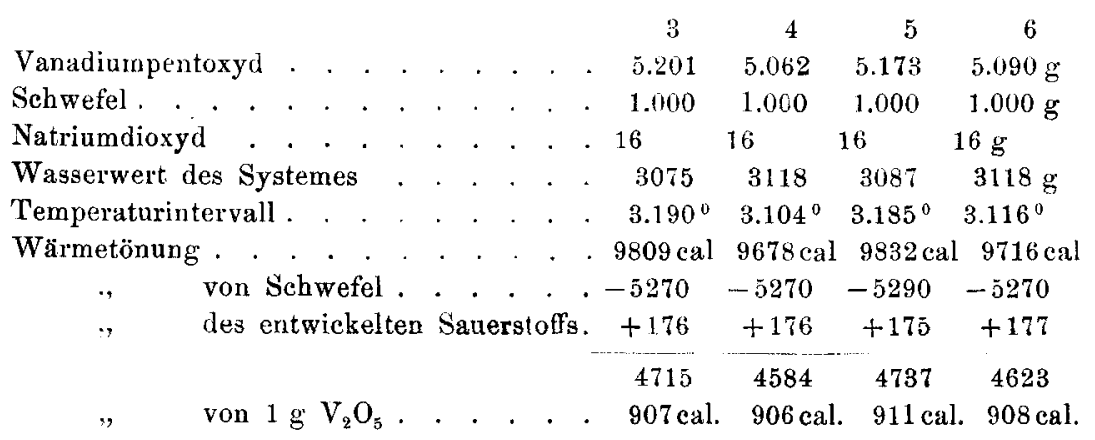

Man fand bei Versuch 4, daß die Verbindungen zum Sammeln des entwickelten Sauerstoffs leckten, weswegen bei 3 und 4 dieselben Korrekturen gemacht wurden wie bei 4 und 5 , da die Bedingungen bei diesen $\nabla$ ersuchen stets im wesentlichen die gleichen waren. Nach den Ergebnissen scheint es, daß die sogenannte amorphe Form von Vanadiumpentoxyd kristallisiert ist; denn wenn sie amorph wäre, sollte die Wärmetönung höher sein als bei der roten kristallisierten Form. Der Mittelwert der letzten beiden Versuche ist 910 cal. Für die Verbindung von 1 Grammolekel Vanadiumpentoxyd mit Natriumoxyd beträgt er 165800 cal.

\section{Vanadiumtrioxyd.}

Bei den folgenden Versuchen kam das Vanadiumtrioxyd $F$ zur Anwendung.

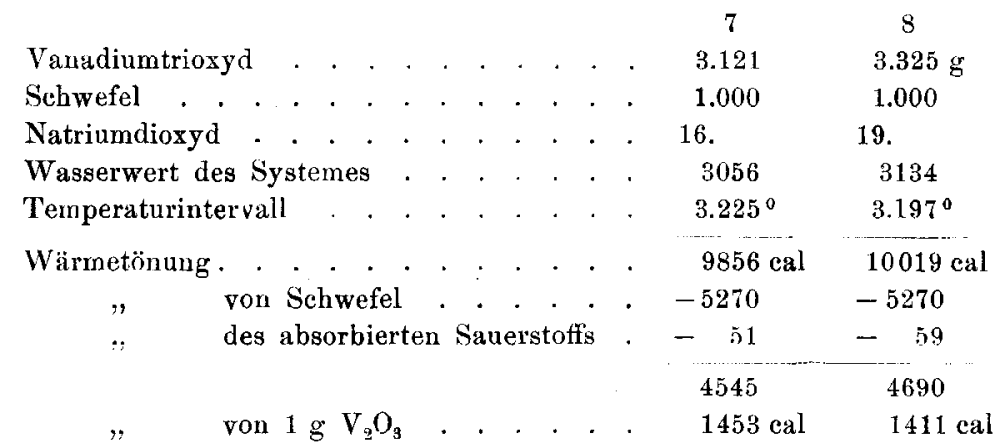

Der Mittelwert 1432 cal., multipliziert mit 150 ergibt $214800 \mathrm{cal}$ für die Reaktionswärme von 1 Grammolekel Vanadiumtrioxyd mit Natriumperoxyd.

Versuch 9. Die direkte Bestimmung der Oxydationswärme des Vanadiumtrioxyds wurde ausgeführt nach dem Verfahren, das man 
angewandt hatte, um die Verbrennungswärme von Titan ${ }^{1}$ zu bestimmen; man benutzte $3 \mathrm{~g}, \mathrm{~F}^{\prime}$, welches nicht vollständig in $\mathrm{V}_{2} \mathrm{O}_{5}$ umgewandelt wurde. Eine gewogene Menge des Verbrennungsproduktes oxydierte man durch Salpetersäure und bestimmte darin das Vanadium. Aus den erhaltenen Zahlen fand man das Atomverhältnis von $V: O$ zu $1: 2.2$. Die beim kalorimetrischen Versuche beobachtete Wärmetönung ist 1664 cal, wovon auf die Baumwolle 558 entfallen, so daB 1104 cal verbleiben. Demnach liefert die Verbrennung von 1 Grammolekel Vanadiumtrioxyd zu Vanadiumtetroxyd und etwas Pentoxyd $\frac{1104}{3} \times 150=55200 \mathrm{cal}$.

\section{Vanadiumtetroxyd.}

Vanadiumtetroxyd stellte man dar durch halbstündiges Erhitzen eines Gemisches äquivalenter Mengen ron Tri- und Pentoxyd in einem ausgepumpten Rohr auf Rotglut. Dieses wurde etwas aufgeblasen, woraus sich ergab, daß geringe Mengen von Sauerstoff abgegeben wurden. Das Produkt war eine koksähnliche Masse. Eine Analyse ergab 61.1\% Vanadium; berechnet für $\mathrm{V}_{2} \mathrm{O}_{4} 61.1 \%$. Vanadiumtetroxyd verbrennt leicht mit Natriumdioxyd ohne Zusatz von Schwefel zu dem Gemisch. Zwei Bestimmungen, bei denen je $4 \mathrm{~g}$ zur Anwendung kamen, lieferten 1084 und 1021 cal. Das Mittel ist 1052 cal oder 174600 für die Reaktion von 1 Gramımolekel Vanadiumtetroxyd mit Natriumdioxyd.

\section{Vanadiumdioxyd.}

Vanadiumdioxyd erhielt man in Kristallen durch Reduktion von Vanadiumoxychlorid, $\mathrm{VOCl}_{3}$, mit Wasserstoff. Benzecios ${ }^{2}$ erhitzte ein Gemisch von Vanadiumpentoxyd und Kalium, entfernte den löslichen Teil mit Wasser und betrachtete das Produkt als inetallisches Vanadium. Roscot ${ }^{3}$ sagt, es wäre ein Gemisch von Oxyden und andererseits, ${ }^{4}$ es wäre das Dioxyd. Gewöhnlich wird in der Literatur angegeben, daB Vanadiumdioxyd nach dem Verfahren von Benzeurus hergestellt werden kann. Doch scheint dies nicht weiter untersucht worden $z u$ sein. Roscos ${ }^{5}$ erbitzte Vanadium-

\footnotetext{
1 Amer. Journ. Sei. (Sill.) 35, 45.

- Pogg. Ann. 22, 3.

3 Phil. Trans. 159, 687.

4 lbid. 690 .

"Roscoe und Schorremmers ,Treatise on Chemistry", 4. Aufl, II, 902.
} 
pentoxyd und Natrium in einem geschlossenen Eisentiegel, wusch das Produkt und erhielt Vanadiumtrioxyd, gemischt mit etwas höherem Oxyd. Ich selbst versuchte die Reaktion mit Natrium in der folgenden Weise: $25 \mathrm{~g}$ Vanadiumtrioxyd und $15 \mathrm{~g}$ Natrium wurden in einem schweren, mit aufgeschraubtem Deckel verschlossenen Messingzylinder erhitzt. Die Reaktion war sehr heftig und der Deckel wurde zum Teil geschmolzen. Das verbleibende metallische Natrium entfernte man durch Alkohol und den zurückbleibenden Rest wusch man mit Wasser, Alkohol und Äther und trocknete ihn im Vakuum. Er wog $15 \mathrm{~g}$. Sodann wurden diese $15 \mathrm{~g}$ und Natrium in einem Eisenzylinder erhitzt, bis das Natrium fast aufhörte, über der Schraubkappe $z u$ brennen. Beim Öffnen des erkalteten Zylinders entzündete sich der Inhalt oben und wurde dann sogleich in Alkohol gebracht, aber es entwickelte sich nur wenig Wasserstoff. Das erhaltene schwarze Pulver wusch man mit Wasser, digerierte es mit verdünnter heißer Chlorwasserstoffsäure, die etwas daron löste, wusch schließlich mit Wasser und trocknete. Man fand bei der Analyse, daß fast reines Vanadiumtrioxyd vorhanden war. Ohne Zweifel reduziert Natrium das Vanadiumtrioxyd, aber es ist nicht für die Reduktion so gut geeignet wie Kalium, welches nicht so heftig reagiert, wahrscheinlich weil es bei niedrigerer Temperatur destilliert als Natrium.

Die folgenden Präparate wurden in einem Eisenzylinder hergestellt, dessen Inhalt nur etwas größer war, als die Menge des Gemisches. Man brächte das Kalium auf den Boden des Zylinders und das Vanadiumtrioxyd darüber in Form eines voluminösen Pulvers, mit Ausnahme eines Falles, der später besprochen wird. Den Zylinder hielt man auf Rotglut, bis kein Kalium mehr über der Schraubenkappe brannte und dann kühlte man ihn schnell in Wasser. Das schwarze Produkt wurde mit kaltem Wasser digeriert und gewaschen, und im Vakuum über Schwefelsäure getrocknet. Eine gewogene Menge des Produktes verwandelte man für die Bestimmung des Vanadiums in Pentoxyd.

Präparat „H“. $7 \mathrm{~g}$ Vanadiumtrioxyd und $5 \mathrm{~g}$ Kalium. Das Produkt wog $2 \mathrm{~g}$ und enthielt $75.9 \%$ Vanadium; $\mathrm{V}_{2} \mathrm{O}_{2}$ enthält $76.1 \%$.

Präparat „I". $12 \mathrm{~g}$ Vanadiumtrioxyd wurden in den Zylinder hineingepreßt, dazu kamen $7 \mathrm{~g}$ Kalium; das Produkt wog $7 \mathrm{~g}$ und enthielt $72.5 \%$ Vanadium. Gleiche Molekel $\mathrm{V}_{2} \mathrm{O}_{2}$ und $\mathrm{V}_{2} \mathrm{O}_{3}$ enthalten $72.1 \%$. Eine Bestimmung der Reaktionswärme von "I" mit Natriumperoxyd zeigte, daB die Oxyde nicht verbunden waren. 
Präparat „J“. $25 \mathrm{~g}$ Vanadiumtrioxyd und $15 \mathrm{~g}$ Kalium. Der Zylinder hatte leider einen Fehler und öffnete sich beim Abkühlen unter Wasser, so daB das Produkt mit Eisenschuppen gemischt wurde. Das schwarze Pulver, das man durch Schlemmen abtrennte, gab bei der Analyse $76.3 \%$ Vanadium, einschlieBlich einer Spur Eisen. Beim Auflösen in Chlorwasserstoffsäure wurde etwas Wasserstoff entwickelt.

Präparat „K $\because .34 \mathrm{~g}$ Vanadiumtrioxyd und $23 \mathrm{~g}$ Kalium. Es befand sich ein kleines Loch oben im Zylinder, das mit einem Pflock verschlossen wurde, wenn das Kalium aufhörte, aus dem Loch herauszubrennen. Den Zylinder kühlte man unter Wasser. Ausbeute $15 \mathrm{~g}$ mit einem Vanadiumgehalt von $76.3 \%$. Die wässerige Lösung war rötlich, verlor aber ihre Farbe beim Stenen, woraus hervorging, dab ein Vanadit ${ }^{1}$ vorhanden war, das sich langsam in Vanadat verwandelte. Die Reaktion von Kalium mit Vanadiumtrioxyd verläuft offenbar folgendermaBen:

$$
\begin{aligned}
\mathrm{V}_{3} \mathrm{O}_{3}+2 \mathrm{~K} & =\mathrm{V}_{2} \mathrm{O}_{2}+\mathrm{K}_{2} \mathrm{O} \\
\mathrm{V}_{2} \mathrm{O}_{3}+\mathrm{K}_{2} \mathrm{O} & =\mathrm{K}_{2} \mathrm{~V}_{2} \mathrm{O}_{4},
\end{aligned}
$$

da nach diesen Gleichungen die Ausbeute aus $34 \mathrm{~g}$ Vanadiumtrioxyd $15.1 \mathrm{~g}$ Dioxyd ist, während man $15 \mathrm{~g}$ erhielt. Die erste Gleichung stellt eine endotherme Reaktion dar, da die Oxydationswärme von Vanadiumdioxyd zu Trioxyd 145000 cal und die der Oxydation von Kalium 97000 cal beträgt. Aber die Reaktion des Systemes ist exotherm und die von $\mathrm{V}_{2} \mathrm{O}_{3}+\mathrm{K}_{2} \mathrm{O}$ ist größer als $97000 \mathrm{cal}$. Ein Teil von "K" wurde in Wasser gebracht in einem Rohr, durch das ein Wasserstoffstrom geleitet wurde, um die Luft zu verdrängen. Zuerst kochte man das Wasser, dann setzte man Salzsäure zu. Die Substanz löste sich mit grüner Farbe obne Gasentwickelung.

Ich verstehe diese Reaktion nicht, denn $\mathrm{V}_{2} \mathrm{O}_{2}$ sollte $\mathrm{VCl}_{2}$ liefern, welches unter Bildung von $\mathrm{VCl}_{3}$ Wasserstoff entwickeln müßte.

Bei den folgenden kalorimetrischen Versuchen kam bei Nr. 11 das Prüparat „,H“, bei den Versuchen 12 und 13 das Präparat ,K“ zur Anwendung.

2 Bestimmungen wurden ausgeführt mit dem nicht sehr reinen Präparat „J“, welches $2810 \mathrm{cal}$ und $2799 \mathrm{cal}$ lieferte. Die so mit

1 Anmerkung des Übersetzers: Als Vanadite bezeichnet man gewöhnlich Salze des vierwertigen Vanadiums vom Typus $R_{2}{ }^{\prime}\left(V_{4} O_{9}\right)$ aq. 


\begin{tabular}{|c|c|c|c|c|c|c|}
\hline & & & & 11 & 12 & 13 \\
\hline Vanadiumdioxyd & d. & & • & 1.7374 & 2.002 & $2.104 \xi$ \\
\hline Natriumdioxyd & . . . & . . . & . & 6 & 8.3 & $8.6 \mathrm{gr}$ \\
\hline Wasserwert des & s Systemes. & 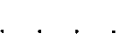 & . & 2898 & 3093 & $3067 \mathrm{~g}$ \\
\hline Temperaturinter & ervall. . . &. & . & $1.565^{\circ}$ & $1.589^{\circ}$ & $1.677^{\circ}$ \\
\hline Värmetönung . & $\cdot$ & $\cdot \cdot \cdot$ & . . & 4535 & 4915 & 5143 \\
\hline $\mathrm{v}$ & von Eisen . . & . . . . & . . & -40 & -40 & -40 \\
\hline$"$ & des entwickelten & Sauersto & offs & +109 & +220 & +230 \\
\hline $\mathrm{v}$ & von $1 \mathrm{~g} \mathrm{~V}_{\mathrm{g}} \mathrm{O}_{\mathrm{q}}$ & $\cdot$ & & $\begin{array}{l}4604 \\
2650 \mathrm{cal}\end{array}$ & $\begin{array}{l}509 \overline{0} \\
254 \tilde{\mathrm{cal}}\end{array}$ & $\begin{array}{l}5333 \\
2535 \mathrm{ca}\end{array}$ \\
\hline
\end{tabular}

verschiedenen Präparaten erhaltenen Resultate weichen etwas voneinander ab; die von 12 und 13 sind als die besten zu betrachten und geben 340000 cal für die Wärmeentwickelung von 1 Grammmolekel Vanadiumdioxyd bei der Reaktion mit Natriumperoxyd.

Versuch 14. $1.173 \mathrm{~g}$ von " $\mathrm{K}$ " $-\nabla_{2} \mathrm{O}_{2}$ wurden, wie bei Versuch 9 beschrieben, in Sauerstoff verbrannt. Der gröBte Teil des Verbrennungsproduktes bestand aus einer Kugel, die äußerlich die Farbe des Pentoxyds hatte, sonst aber schwarz und kristallinisch war. Das Atomverhältnis von Vanadium zu Sauerstoff darin war 1 :2.2.

Zusammenstellung der Resultate.

\begin{tabular}{|c|c|c|c|c|c|}
\hline $\begin{array}{l}2 \mathrm{~V}+5 \mathrm{O}=\mathrm{V}_{2} \mathrm{O}_{5}+. \text {. wahrseheinli } \\
\mathrm{V}_{2} \mathrm{O}_{5}+3 \mathrm{Na}_{2} \mathrm{O}=3 \mathrm{Na}_{2} \mathrm{O} \cdot \mathrm{V}_{2} \mathrm{O}_{5}+.\end{array}$ & & & & & 165800 \\
\hline $\begin{array}{l}\mathrm{V}_{2} \mathrm{O}_{3}+2 \mathrm{Na}_{2} \mathrm{O}_{2}+\mathrm{Na}_{2} \mathrm{O}=3 \mathrm{Na}_{2} \mathrm{O} \cdot \mathrm{V}_{2} \mathrm{O}_{5} \\
2 \mathrm{Na}_{2} \mathrm{O}+2 \mathrm{O}=2 \mathrm{Na}_{2} \mathrm{O}_{2}+\ldots\end{array}$ & + & & . & . & $\begin{array}{r}214800 \\
38800\end{array}$ \\
\hline $\begin{array}{l}\mathrm{V}_{2} \mathrm{O}_{3}+2 \mathrm{O}+3 \mathrm{Na}_{2} \mathrm{O}=3 \mathrm{Na}_{2} \mathrm{O} \cdot \mathrm{V}_{2} \mathrm{O}_{5}+ \\
\mathrm{V}_{2} \mathrm{O}_{5}+3 \mathrm{Na}_{2} \mathrm{O}=3 \mathrm{Na}_{2} \mathrm{O} \cdot \mathrm{V}_{2} \mathrm{O}_{5}+\end{array}$ & . $\cdot$ & . & . & • & $\begin{array}{l}253600 \\
165800\end{array}$ \\
\hline $\mathrm{V}_{2} \mathrm{O}_{3}+2 \mathrm{O}=\mathrm{V}_{2} \mathrm{O}_{5}+\ldots . . .$. & . . & . & . & . & 87800 \\
\hline$a_{2} \mathrm{O}_{2}+2 \mathrm{Na}_{2} \mathrm{O}=3 \mathrm{Na}_{2} \mathrm{O} \cdot \mathrm{V}_{2} \mathrm{O}_{\overline{2}}$ & + . & & . & . & 174600 \\
\hline $\mathrm{Na}_{2} \mathrm{O}+\mathrm{O}=\mathrm{Na}_{2} \mathrm{O}_{2}+\ldots$ & & & 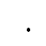 & . & 19400 \\
\hline $\mathrm{V}_{2} \mathrm{O}_{4}+\mathrm{O}+3 \mathrm{Na}_{2} \mathrm{O}=3 \mathrm{Na}_{2} \mathrm{O} \cdot \mathrm{V}_{2} \mathrm{O}_{5}+$ & . & . & 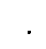 & 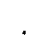 & 194000 \\
\hline $\mathrm{V}_{2} \mathrm{O}_{5}+3 \mathrm{Na}_{3} \mathrm{O}=3 \mathrm{Na}_{2} \mathrm{O} \cdot \mathrm{V}_{2} \mathrm{O}_{5}+\ldots$ & . & . & . & . & 165800 \\
\hline $\mathrm{V}_{2} \mathrm{O}_{4}+\mathrm{O}=\mathrm{V}_{2} \mathrm{O}_{5}+\ldots$ & - & . & . & t. & 28200 \\
\hline $\begin{array}{l}\mathrm{V}_{2} \mathrm{O}_{2}+3 \mathrm{Na}_{2} \mathrm{O}_{2}=3 \mathrm{Na}_{2} \mathrm{O} \cdot \mathrm{V}_{2} \mathrm{O}_{5}+. \\
3 \mathrm{Na}_{2} \mathrm{O}+3 \mathrm{O}=3 \mathrm{Na}_{2} \mathrm{O}_{2}+.\end{array}$ & & & . & . & $\begin{array}{r}340000 \\
58200\end{array}$ \\
\hline $\mathrm{V}_{2} \mathrm{O}_{2}+3 \mathrm{O}+3 \mathrm{Na}_{2} \mathrm{O} \cdot \mathrm{V}_{2} \mathrm{O}_{5}+\ldots$ & 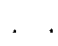 & . & . & . & 398200 \\
\hline $\mathrm{Na}_{2} \mathrm{O}=3 \mathrm{Na}_{2} \mathrm{O} \cdot \mathrm{V}_{2} \mathrm{O}_{5}+$ & & . & . & . & 165800 \\
\hline $30=V_{2} O_{5}+\ldots . .$. & . & . & . & . & 232400 \\
\hline$=\mathrm{V}_{\mathrm{g}} \mathrm{O}_{8}+$ & & & . & & 140600 \\
\hline $\mathrm{O}=\mathrm{V}_{2} \mathrm{O}_{4}+$ & & & . & - & 204200 \\
\hline $\mathrm{V}_{2} \mathrm{O}_{3}+\mathrm{O}=\mathrm{V}_{2} \mathrm{O}_{4}+$ & & - & . & & $59600 \mathrm{cal}$ \\
\hline
\end{tabular}

Experimentelles Resultat.

t. Abgeleitetes Resultat. 
Die beobachtete Wärmetönung, vermindert um die der Baumwolle, betrug 1819 cal. Demnach ist die Wärmetönung der Verbrennung von $\mathrm{V}_{2} \mathrm{O}_{2}$, hauptsächlich zu $\mathrm{V}_{2} \mathrm{O}_{4}, \frac{1819}{1.173} \times 134=208000 \mathrm{cal}$.

Die Wärmetönung von $\mathrm{V}_{2} \mathrm{O}_{3}+\mathrm{O}$, die sich beim Verbrennen in Sauerstoff (Versuch 9) fand, betrug 55.2 cal; aber das Verfahren war nicht befriedigend, denn bei einem anderen Versuch verbrannte nur wenig von dem Vanadiumtrioxyd. Vanadiumdioxyd dagegen verbrannte gut und gab in Versuch 14 für die Reaktion $\mathrm{V}_{2} \mathrm{O}_{2}$ $2 \mathrm{O}=\mathrm{V}_{2} \mathrm{O}_{4}=208000$ cal. Dies ist etwas hoch, da etwas Pentoxyd gebildet wurde; aber der Wert bestatigt die nach der Natriumdioxydmethode gefundene Zahl.

Bei diesen Resultaten fällt die große Oxydationswärme von Vanadiumdioxyd auf; auch zeigen die mit unreinem Metall erhaltenen Resultate, daB bei der Bindung der beiden ersten Sauerstoffatome an Vanadium nicht ein gröBerer Wärmeeffekt auftritt, als beim dritten. Nehmen wir an, daB sie denselben Effekt geben, so haben wir bei $2 \mathrm{~V}+5 \mathrm{O}=\mathrm{V}_{2} \mathrm{O}_{5}+520000 \mathrm{cal}$, und gefunden wurden nur 441000 cal.

Nacbdem die vorstehenden Zeilen geschrieben waren, erschien die Mitteilung von RufF und Mar'rin ${ }^{1}$ über Vanadium. Diese finden für die Bildungswärme von Vanadiumpentoxyd 250500 cal, ein Wert, der viel kleiner ist, als die hier mitgeteilten Resultate erwarten lassen. Z. B. deutet die Wärmetönung der Reaktion $\mathrm{V}_{2} \mathrm{O}_{2}+$ $20=204000 \mathrm{cal}$ nach der Natriumdioxydmethode, die noch durch Verbrennung in Sauerstoff bestätigt wurde, deutlich auf eine Wärmetönung von mehr als 400000 cal für die Bildung von Vanadiumpentoxyd. Denn es muB angenommen werden, daß die beiden ersten Sauerstoffatome ebensoviel Wärme entwickeln wie die beiden letzten.

Im Hinblick auf die Arbeit von RuFF und Martis schien es wünschenswert, Vanadium in Sauerstoff zu verbrennen. Das beste Material, das zur Verfügung stand, war Präparat „C“, das $89.7 \%$ Vanadium sogleich nach der Herstellung und $88.6 \%$ Monate später enthielt. Es war darin noch eine Spur Magnesium. Die Bestimmungen wurden wie in Versuch 9 ausgeführt und der Sauerstoff kam mit 15 Atmosphären Druck zur Anwendung. Das Produkt der Verbrennung bildete eine Kugel auf dem Boden der Bombe, während

1 Zcitsehr. angew. Chem. 25, 49. 
kleine Tröpfchen und ein dunkelrotes Pulver am Deckel hingen. Die Temperatur der Verbrennung war hoch und in einem Falle war die schwere Platinelektrode geschmolzen. Die folgende Tabelle enthält die Einzelheiten der Bestimmung.

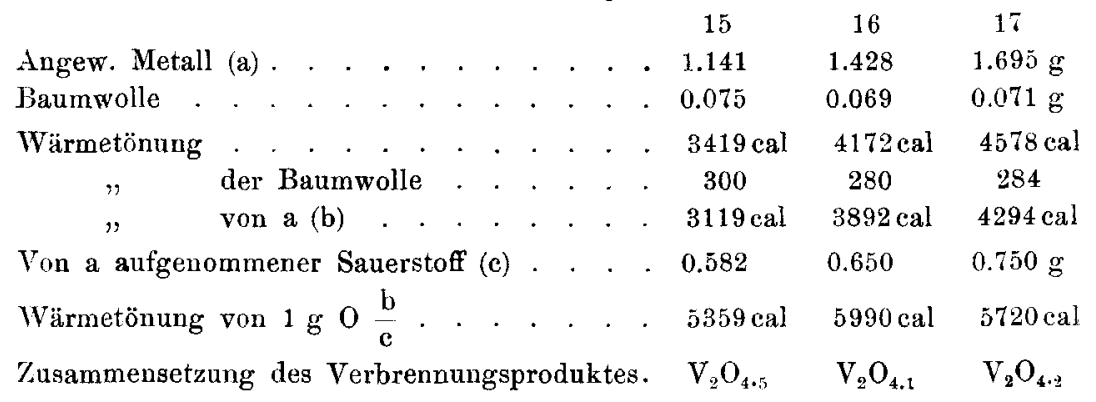

Der Mittelwert für die Verbindungswärme von $1 \mathrm{~g}$ Sauerstoff mit dem oxydierten Metall beträgt 5690 cal., dies liefert für 4.2 Grammatome Sauerstoff $5690 \times 16 \times 4.2=382000$ cal. Dieses ist die Wärmetönung der Oxydation eines Gemisches von Vanadium und Vanadiumdioxyd, das eine groBe Menge von dem letzteren enthält. Demnach ist das Resultat viel niedriger, als bei reinem Metall. Es ist unmöglich, für das Oxyd eine Korrektur anzubringen, die in jedem Falle groß werden würde.

\section{Uran.}

Es ist bemerkenswert, daß keine thermische Daten über Uranverbindungen vorhanden sind, da sowohl das Metall wie auch das Dioxyd gut in Sauerstoff verbrennen. Die Bildungswärme des Dioxyds hat man wegen seiner schwierigen Reduzierbarkeit als groB betrachtet. Professor B. B. BoLTwood lieferte uns freigebig eine reichliche Menge von reinem Uranylnitrat für die Untersuchung.

Metallisches Uran wurde hergestellt nach dem Verfabren von Morssan,${ }^{1}$ das in einigen Einzelheiten abgeändert wurde. Auf dem Boden eines Stahlzylinders brachte man $30 \mathrm{~g}$ Natrium und hierauf $40 \mathrm{~g}$ eines Gemisches des Doppelsalzes $\mathrm{UCl}_{4} .2 \mathrm{NaCl}$ mit etwa 2 Äquivalenten Natriumchlorid in kleinen Stücken. Das Natriumchlorid war bei der Herstellung des Doppelsalzes im Überschuß vorhanden. Den Zylinder verschloß man mit einer losen Schraubkappe und erhitzte ihn 15 Minuten auf Rotglut. Der gröBte Teil des überschüssigen Natriummetalles verflüchtigte sich und verdampfte

1 Compt. rend. 122, 1088. 
durch den aufgeschraubten Deckel. Der geringe, im Zylinder verbleibende Rest wurde mit Alkohol entfernt. Das Natriumchlorid löste man in entlüftetem kaltem Wasser, und dann wusch man das Metall mit Wasser, Alkohol und Äther und trocknete es im Vakuum über Schwefelsäure. Das Metall befand sich dann in Form einer zerreiblichen Masse. Es wurde pulverisiert und durch ein Millimetersieb hindurchgetrieben. Der gröbere Teil entzündete sich beim Zerreiben in einem Mörser. Zur Analyse löste man das Metall in Königswasser und fällte das Hydroxyd mit Ammoniak.

$$
\begin{aligned}
& 0.3222 \mathrm{~g} \text { gaben } 0.3766 \mathrm{~g} \mathrm{U} \mathrm{U}_{2}=99.2 \mathrm{O}_{8} \mathrm{U}, \\
& 0.9001 \mathrm{~g}, \quad 1.01+4 \mathrm{~g} \mathrm{UO}=99.4 \%_{0}^{\circ} \mathrm{U} .
\end{aligned}
$$

Das Metall enthielt eine Spur Natrium. Die zweite Analyse wurde 9 Tage nach der ersten ausgeführt; die Resultate zeigen, daB das Metall sich in dem Exsikkator, wo es aufgehoben wurde, nicht oxydierte. Es nahm auch nicht an Gewicht zu, als es eine Zeitlang an der Luft lag; die braune Decke von Oxyd wirkte als Schutz. Eine andere Metallprobe, die man in der angegebenen Weise herstellte, gab bei der Analyse folgende Zahlen:

$$
\begin{aligned}
& 1.0378 \mathrm{~g} \text { Metall : } 1.543 \mathrm{~g} \mathrm{UO}_{2}=98.07 \% \mathrm{U} \text {, } \\
& 1.0336 \mathrm{~g} \quad, \quad 1.1482 \mathrm{~g} \mathrm{UO}_{2}=97.93 \% \mathrm{C} \text {. }
\end{aligned}
$$

Das Metall enthielt $0.07 \%$ Natrium. Beide Uranproben waren frei von Chlor und Kohle und beide lösten sich langsam in Chlorwasserstoffsäure und hinterlieBen einen geringen Rückstand, welcher eine in Königswasser lösliche Uranverbindung darstellte. Das Metall war vollständig löslich in Salpetersäure.

Nach Fertigstellung der kalorimetrischen Untersuchung wurde eine dritte Uranprobe hergestellt, wobei man das Metall in geschmolzenen Stücken und zerreiblichen Klumpen erhielt. Von diesen sonderte man Körner von $1 / 2-1 \mathrm{~mm}$ Durchmesser ab, deren Analyse $99.7 \% \mathrm{U}$ und $0.09 \%$ Natrium ergab. Diese Körner verbrannten nur unvollständig mit Natriumdioxyd in der Bombe und gaben zu niedrige Resultate. Deswegen wurden keine weiteren kalorimetrischen Bestimmungen mit dieser Uranprobe angestellt.

Die ersten 5 Versuche wurden ausgeführt mit dem Metall, das $99.4 \%$ Uran enthielt, und die letzten beiden mit dem Muster von $98 \%$. Aus den Resultaten sieht man, dab $1 \mathrm{~g}$ Sauerstoff bei der Bildung von $\mathrm{U}_{3} \mathrm{O}_{3}$ eine Wärmeentwickelung von $6590 \mathrm{cal}$ ergibt. Welches die Zusammensetzung des das Metall bedeckenden Oxyds ist, weiB man nicht, wahrscheinlich aber ist es ein niedriges Oxyd 
und demnacb kann die Wärmetönnng seiner Bildung etwas höher sein. Wie dies aber auch sein mag, das Produkt von $6590 \mathrm{mit}$ dem Sauerstoffgehalt des angewandten Metalles wurde zu der beobachteten Wärmetönung bei den folgenden Versuchen zugezählt.

\begin{tabular}{|c|c|c|c|c|c|c|c|}
\hline & 1 & 2 & 3 & 4 & 5 & 6 & 7 \\
\hline ngew. Metall. & 1.7294 & 2.1944 & 2.1136 & 2.8266 & 4.7255 & 5.0623 & $4.4108 \mathrm{~g}$ \\
\hline Zus. des | U (a) & 1.7190 & 2.1812 & 2.1009 & 2.8096 & 4.6971 & 4.9611 & $4.3226 \mathrm{~g}$ \\
\hline Metalles $\int \mathrm{O}(\mathrm{b})$ & 0.0104 & 0.0132 & 0.0127 & 0.0170 & 0.0284 & 0.1012 & $0.0882 \mathrm{~g}$ \\
\hline Baumwolle & 0.0899 & 0.1005 & 0.1012 & 0.0928 & 0.0822 & 0.0830 & $0.0795 \mathrm{~g}$ \\
\hline Wasserwert d.syst. & 1352 & 1343 & 1466 & 1329 & 2979 & 2995 & 2867 \\
\hline Temperaturinterv. & $1.601^{\circ}$ & $2.119^{\circ}$ & $1.916^{\circ}$ & $2.704^{\circ}$ & $1.857^{\circ}$ & $1.836^{\circ}$ & $1.674^{\circ}$ \\
\hline Wärmetönung . & $2168 \mathrm{cal}$ & $2846 \mathrm{cal}$ & $2809 \mathrm{cal}$ & $3594 \mathrm{cal}$ & $5532 \mathrm{cal}$ & $5549 \mathrm{cal}$ & $4803 \mathrm{cal}$ \\
\hline Baumwolle. & -363 & -404 & -405 & -374 & -329 & -335 & -321 \\
\hline 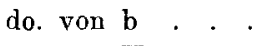 & +68 & +87 & $+\varepsilon$ & +112 & +187 & +666 & +581 \\
\hline$(c)$ & 1873 & 2529 & 2488 & 3332 & 5390 & 5830 & 5063 \\
\hline
\end{tabular}

do. v. $1 \mathrm{~g}$ Uran $\underset{\mathrm{a}}{\mathrm{e}} 1089 \mathrm{cal} 1169 \mathrm{cal} 1184 \mathrm{cal} 1186 \mathrm{cal} 1147 \mathrm{cal} 1175 \mathrm{cal} 1176 \mathrm{cal}$ Zus. des Verbren$\begin{array}{llllllll}\text { nungsproduktes } & \mathrm{U}_{3} \mathrm{O}_{7.07} & \mathrm{U}_{4} \mathrm{O}_{7.83} & \mathrm{U}_{3} \mathrm{O}_{8.04} & \mathrm{U}_{3} \mathrm{O}_{8.00} & \mathrm{U}_{\mathbf{3}} \mathrm{O}_{7.70} & \mathrm{U}_{9} \mathrm{O}_{7.88} & \mathrm{U}_{3} \mathrm{O}_{7.94}\end{array}$

Schließt man die Resultate von 1 und 5 aus, bei denen die Oxydation weniger vollständig war, als bei den anderen, so ist das Mittel 1178 cal. Für die Bildungswärme von $\mathrm{U}_{3} \mathrm{O}_{8}$ haben wir dann $1178 \mathrm{cal} \times 238.5 \times 3=842900 \mathrm{cal}$ bei konstantem Volumen und 845200 cal bei konstantem Druck.

Da das Metall mit 98\% Uran nicht vollständig als ein für die Verbrennung mit $\mathrm{Na}_{2} \mathrm{O}_{2}$ brauchbares Pulver vorlag, wurden die zerreiblichen Sticke zerbrochen und nur das feine Pulver benutzt. Man fand nun, daß es $97.5 \%$ Uran enthielt. Eine Korrektur wurde wie vorher angebracht wegen des Sauerstoffgehaltes des Metalles bei der Berechnung der folgenden Beobachtungen:

Angew. Metall . . . . . . . . . . 6.014

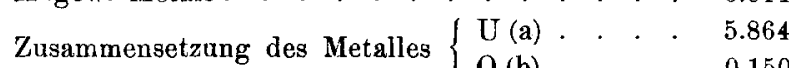

Wasserwert des Systemes. . . . . . . . . 2914

Temperaturintervall . . . . . . . . . . . $2.502^{\circ}$

Wärmetönung
8

9

$7.060 \mathrm{~g}$

6.884

0.176

2932

$2.930^{\circ}$

$8591 \mathrm{cal}$

$-40$

$+206$

$+1160$

$8365 \quad 9911$

$1426 \mathrm{cal} \quad 1440 \mathrm{cal}$ 
Der Mittelwert $1433 \times 233.5=341800 \mathrm{cal}$ ist die Realtionswärme von 1 Grammatom Uran mit Natriumdioxyd.

\section{Verbindungswärme von Uranioxyd mit Natriumoxyd.}

Uranioxyd wurde hergestellt durch vorsichtiges Erhitzen von Uranylnitrat in Platin, bis die Masse wieder fest wurde, und weiteres Erhitzen in einem Glaskolben auf $400^{\circ}$, bis das Gewicht des Oxyds konstant blieb. Das Präparat erwies sich als frei von Nitrat und

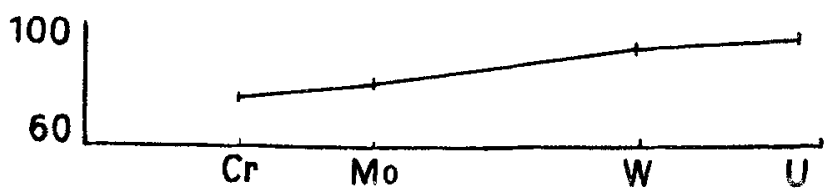

lieferte fast die theoretische Menge von $\mathrm{U}_{3} \mathrm{O}_{8}$. Ein anderes Präparat von Uranioxyd wurde bei den Versuchen 12 und 13 benutzt. Die kalorimetrischen Bestimmungen ergaben das folgende:

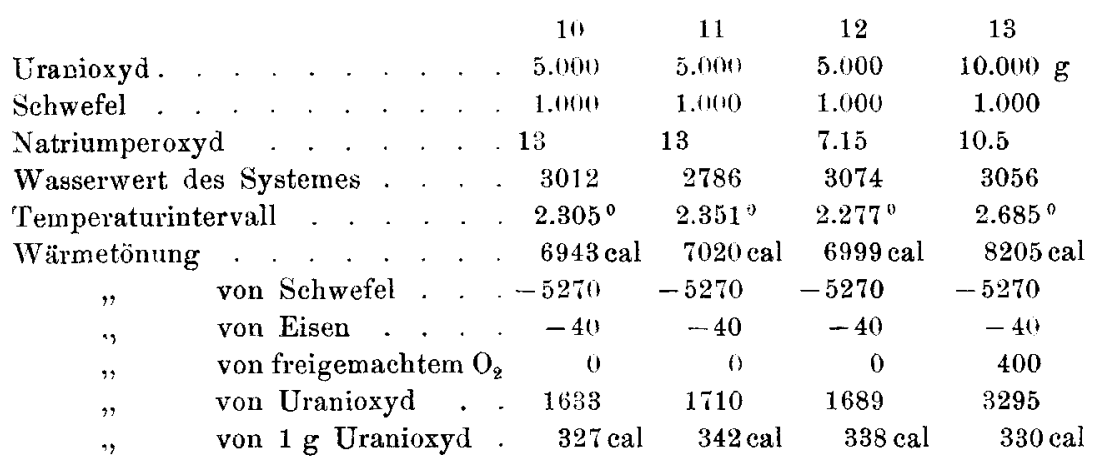

Deimnach ist die Wärmetönung von $\mathrm{Na}_{2} \mathrm{O}+\mathrm{UO}_{3}$ im Mittel $334 \times 286.5=96100 \mathrm{cal}$.

Es ist gezeigt worden, ${ }^{1}$ daB die Reaktionswärme von $\mathrm{Na}_{2} \mathrm{O}+\mathrm{RO}_{3}$ regelmäBig mit den Atomgewichten zunimmt, wenn unter $\mathrm{R}$ Chrom, Molybdän und Wolfram verstanden wird. Uran fällt etwas unter die Linie, die die Verlängerung der Verbindungslinie der 3 anderen Werte bildet, wie in der Figur gezeigt ist, in der die Atomgewichte als Abszissen und die Wärmetönungen als Ordinaten gezeichnet sind.

Das Uran bildet Peruranate; deswegen entsteht die Frage, ob das Uranoxyd in der Schmelze mit Natriumperoxyd Peruranate liefern kann. Die kalorimetrischen Ergebnisse zeigen, daß nur

${ }^{1}$ Amer. Jown. Sri. (Sill.) 299, 490. 
Natriumuranat entsteht, da bei Versuch 12, wo die vorbandene Natriumdioxydmenge nur ausreichte, um Natriumuranat zu bilden die Wärmetönung ebenso groß war, wie bei den anderen Versuchen mit einem ÜberschuB von Dioxyd. Alle Schmelzen in der Bombe waren orangerot. Die Schmelzen von 10 und 11 lösten sich zum gröBten Teile in kaltem Wasser unter Entwickelung von Sauerstoff, wobei eine órange oder rötliche Lösung von Peruranat entstand. Diese Lösung wurde allmählich bei Zimmertemperatur und schnell beim Kochen heller und setzte ein wasserhaltiges Gemisch von Uranaten ab, das annähernd die Zusammensetzung $3 \mathrm{Na}_{2} \mathrm{O} .8 \mathrm{UO}_{3}+$ $\mathrm{xH}_{2} \mathrm{O}$ besaB. Die erwähnte Lösung von Peruranat war das Ergebnis der Einwirkung von Wasser auf das Gemisch von Natriumuranat und -dioxyd; denn man fand, daß jenes sich in einer kalten Lösung von Natriumdioxyd unter Bildung von Peruranat löste.

\section{Urandioxyd.}

Urandioxyd wurde hergestellt durch Reduktion des mit etwas grünem Oxyd gemischten Trioxyds durch Wasserstoff bei dunkler Rotglut. Die Verbrennungen mit Natriumdioxyd ergaben folgendes:

\begin{tabular}{|c|c|c|c|c|c|}
\hline & & & 14 & 15 & 16 \\
\hline Urandioxyd . & . . & 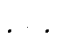 & 5.000 & 10.000 & $5.000 \mathrm{~g}$ \\
\hline Schwefel. &.. & 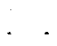 & 1.000 & 1.000 & 1.000 \\
\hline Natriumdioxyd & . & . & 14 . & 14. & 14. \\
\hline Wasserwert des & s Systemes. & . & 2967 & 3101 & 3077 \\
\hline Temperaturinter & rvall . & . . & $2.478^{\circ}$ & $3.035^{\circ}$ & $2.393^{\circ}$ \\
\hline Wärmetönung & . & . & $7352 \mathrm{cal}$ & $9418 \mathrm{cal}$ & $7363 \mathrm{cal}$ \\
\hline " & von Schwefel & . . & -5270 & -5270 & -5270 \\
\hline$"$ & von Eisen & . & -40 & -40 & -40 \\
\hline$"$ & von $\mathrm{UO}_{2}$. & . & 2042 & 4108 & 2053 \\
\hline$"$ & von $1 \mathrm{~g} \mathrm{UO}_{2}$ & . & $408 \mathrm{cal}$ & $411 \mathrm{cal}$ & $410 \mathrm{cal}$ \\
\hline
\end{tabular}

Als Mittel findet man für die Wärmetönung von $\mathrm{UO}_{2}+$ $\mathrm{Na}_{2} \mathrm{O}_{2}=\mathrm{Na}_{2} \mathrm{UO}_{4}$ den Wert $410 \times 270.5=110900 \mathrm{cal}$.

Bei den folgenden Verbrennungen ron Urandioxyd mit Sauerstoff kam dieselbe Dioxydprobe zur Anwendung mit Ausnahme des Versuches 21; bei diesem stammte das Dioxyd von den Reduktionen der höheren Oxyde, die beim Verbrennen des Dioxyds in Sauerstoff erbalten waren.

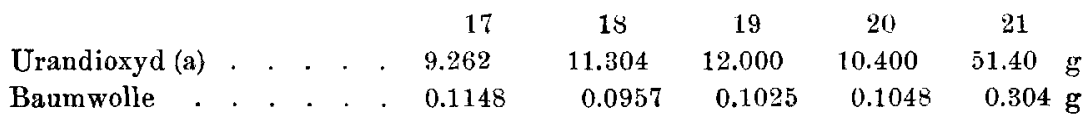


H. G. Mixter:

\begin{tabular}{|c|c|c|c|c|c|}
\hline & 17 & 18 & 19 & 20 & 21 \\
\hline $\begin{array}{l}\text { usammensetzung des Ve } \\
\text { brennungsproduktes }\end{array}$ & $\mathrm{U}_{8} \mathrm{O}_{2.99}$ & $\mathrm{U}_{3} \mathrm{O}_{7.99}$ & $\mathrm{U}_{3} \mathrm{O}_{7.8 \mathrm{I}}$ & $\mathrm{U}_{3} \mathrm{O}_{7.82}$ & $\mathrm{U}_{8} \mathrm{O}_{i .95}$ \\
\hline \multicolumn{6}{|l|}{ Urandioxyd, verbrannt $z u$} \\
\hline $\mathrm{U}_{3} \mathrm{O}_{8}(\mathrm{~b})$ & 8.799 & 11.247 & 10.810 & 9.984 & $50.11 \mathrm{~g}$ \\
\hline Wasserwert des Systemes & 1383 & 1384 & 1281 & 1292 & $2619 \mathrm{~g}$ \\
\hline Temperaturintervall . . & $0.905^{\circ}$ & $1.048^{\circ}$ & $1.132^{\circ}$ & $1.006^{\circ}$ & $2.139^{\circ}$ \\
\hline Wärmetönung & $1251 \mathrm{cal}$ & $1450 \mathrm{cal}$ & $1450 \mathrm{cal}$ & $1300 \mathrm{cal}$ & $5664 \mathrm{cal}$ \\
\hline do. der Baumwolle. & -461 & -386 & -414 & 423 & 1211 \\
\hline do. von $\mathrm{UO}_{2}(\mathrm{c})$. & 790 & 1064 & 1036 & 877 & 4453 \\
\hline do. von $1 \mathrm{~g} \quad \mathrm{UO}_{2} \frac{\mathrm{c}}{\mathrm{b}}$ & $90 \mathrm{cal}$ & $95 \mathrm{cal}$ & $96 \mathrm{cal}$ & $88 \mathrm{cal}$ & $87 \mathrm{cal}$ \\
\hline
\end{tabular}

Für die Oxydationswärme von 1 Grammolekel Orandioxyd zu $\mathrm{U}_{3} \mathrm{O}_{8}$ ergibt sich im Mittel $92 \times 270.5=24900$ cal bei konstantem Volumen und 25100 cal bei konstantem Druck.

\section{Urano-Uranioxyd.}

Dies Oxyd $\mathrm{U}_{3} \mathrm{O}_{8}$, das bei den Versuchen 22 und 23 zur Anwendung kawn, wurde hergestellt durch Erhitzen des Nitrats und weiteres Erhitzen des Rückstandes in Sauerstoff und Ablühlung in demselben Gas. Das Gewicht blieb unverändert, wenn es nochmals in Sauerstoff erhitzt und abgekühlt wurde. Bei Versuch $24 \mathrm{kam}$ das Oxyd zur Anwendung, das durch Verbrennen von Urandioxyd bei Versuch 21 erhalten war und die Zusammensetzung $\mathrm{U}_{3} \mathrm{O}_{7.95}$ hatte.

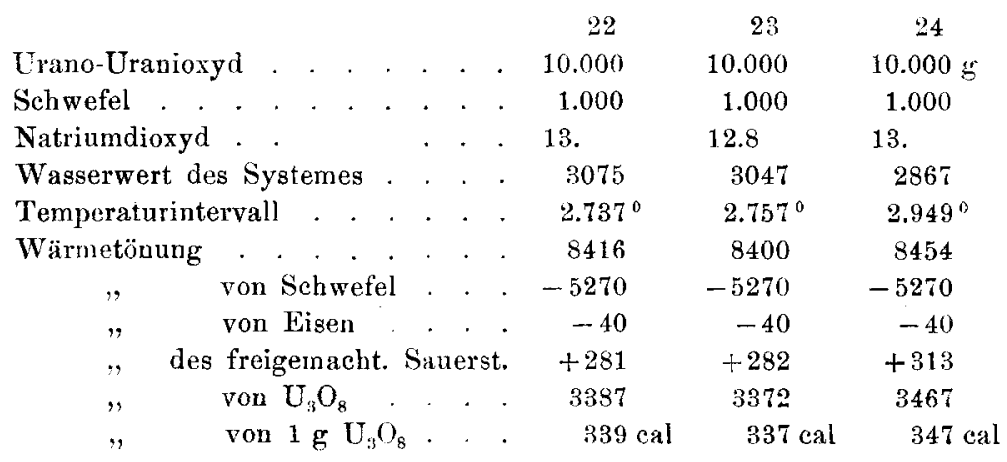

Das letztere Resultat sollte zur Bildung des Endwertes nicht herangezogen werden, da das benutzte Oxyd eine kleine Menge von Dioxyd enthielt. Der Mittelwert der Versuche 22 und 23 ist 338 cal. Für 1 Grammolekel $\mathrm{U}_{3} \mathrm{O}_{8}$ ergeben sich $285100 \mathrm{cal}$. Der Versuch 24 zeigt, daß das bei hohen Temperaturen gebildete $\mathrm{U}_{3} \mathrm{O}_{8}$ 
sich nicht wesentlich von dem sogenannten grünen Oxyd unterscheidet.

Zusammenstellung der Resultate.

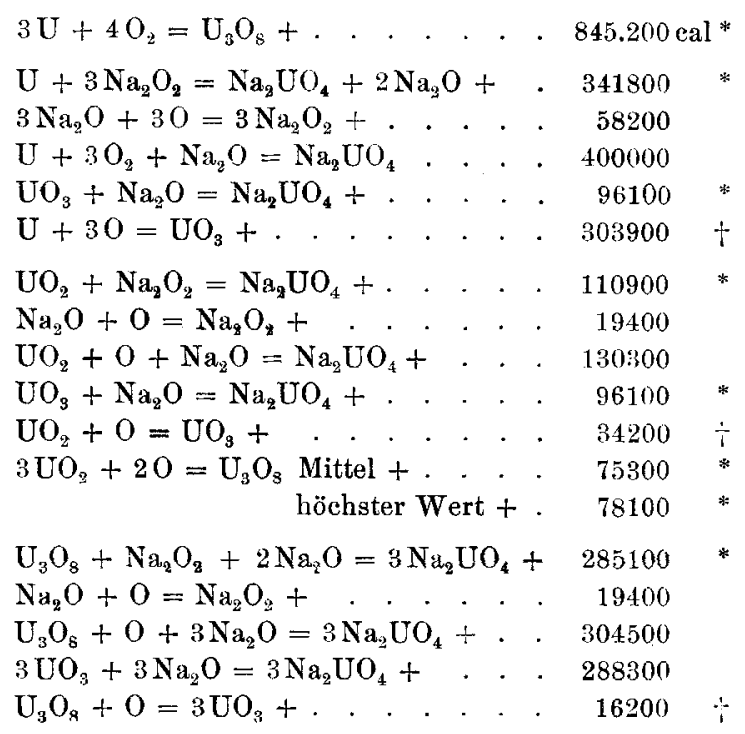

* Experimentell bestimmt. $\div$ Abgeleitet.

Aus den angeführten Daten sind die folgenden Werte abgeleitet für die Verbrennung mit

\begin{tabular}{|c|c|c|c|}
\hline & & Sauerstoff & Natriumdioxyd \\
\hline $3 \mathrm{U}+4 \mathrm{O}_{2}$, & . & . $845200 \mathrm{cal}$ & $895500 \mathrm{cal}$ \\
\hline $\mathrm{U}+\mathrm{O}_{2}$. & . & . 256600 & 269700 \\
\hline $3 \mathrm{UO}_{2}+\mathrm{O}_{2}$ & & 75300 & 86400 \\
\hline
\end{tabular}

Die Resultate mit Natriumdioxyd sind $5 \%$ böher als die mit Sauerstoff bei den beiden ersten Reaktionen und $11 \%$ bei der letzten. Über die Genauigkeit der verschiedenen Zahlen ist folgendes zu sagen: 845200 ist als gut zu betrachten, da es der Mittelwert gut übereinstimmender Zahlen der Verbrennung zweier verschiedener Metallproben mit verschiedenem Sauerstoffgehalt in Sauerstoff ist. Wenn angenommen wird, daB das Oxyd auf dem Metalle $\mathrm{UO}_{2}$ ist anstatt $\mathrm{U}_{\mathbf{3}} \mathrm{O}_{8}$, welches als Basis für die Korrektur bei den Versuchen 1-7 angenommen wurde, so wird das Resultat etwas höher. Die Verbrennung von Urandioxyd in Sauerstoff lieferte Ergebnisse, die prozentisch sehr weit, aber nur 9 cal pro Gramm abweichen. Nimmt man den höchsten Wert, so wird $3 \mathrm{UO}_{2}+\mathrm{O}_{2}=77900$ cal, und hieraus ergibt sich die Zahl für $\mathrm{U}+\mathrm{O}_{2}=255400$ cal. Über

Z. anorg. Chem. Bd. 78 . 
238 W. G. Mixter. Bildungswärmen der Oxyde von Vanadium und Uran.

die Zahlen unter Natriumdioxyd ist zu bemerken, daB die Werte 895500 und 269700 cal abgeleitet sind aus Verbrennungen des Metalles, und daß die Korrektion für 2.5\% Sauerstoff groß ist, während 86400 abgeleitet ist aus der Verbrennung von $\mathrm{UO}_{2}$ und $\mathrm{U}_{3} \mathrm{O}_{8}$. Es könnte scheinen, daß die Ergebnisse mit Natriumdioxyd nicht zuverlässig sind; aber in früheren Mitteilungen ist gezeigt worden, daß die Methode bei Kohle, Schwefel und Titan Resultate liefert, die mit den Werten, welche man beim Verbrennen in Sauerstoff erhält, eng übereinstimmen. Zum Schluß ist noch zu bemerken, daB sich für die hohen Werte mit Natriumdioxyd keine Erklärung gefunden hat.

New Haven, U.S.A., The Sheffield Chem. Laboratory of Yale University.

Bei der Redaktion eingegangen am 17. Juli 1912. 